\title{
Acute Limb Ischemia: Surgical Thromboembolectomy and the Clinical Course of Arterial Revascularization at Ankle
}

\author{
Ha Song Shin, MD ${ }^{1} \quad$ Kyu-Hyouck Kyoung, MD ${ }^{1} \quad$ Byoung Jo Suh, MD ${ }^{1} \quad$ Si-Youl Jun, MD² \\ Jong Kwon Park, MD ${ }^{1}$
}

${ }^{1}$ Department of Surgery, Haeundae Paik Hospital, College of Medicine, Inje University, Haeundae-Gu, Busan, Republic of Korea

2 Department of Surgery, Samsung Changwon Hospital,

Sungkyunkwan University School of Medicine, Changwon, Republic of Korea

Int J Angiol 2013;22:109-114.

\begin{abstract}
Address for correspondence Jong Kwon Park, MD, Department of Surgery, Haeundae Paik Hospital, College of Medicine, Inje University, 1435 Jwa-Dong, Haeundae-Gu, Busan, Republic of Korea 612-080 (e-mail: jkpark@paik.ac.kr).
\end{abstract}

For acute limb ischemia, there are generally three treatment options: the catheter-directed thrombolysis, anticoagulation with observation, and surgical thromboembolectomy. ${ }^{1-5}$ Catheter-directed thrombolysis is less invasive than surgical thromboembolectomy, and is known to be effective for the treatment of acute limb ischemia. ${ }^{1,2}$ However, catheter-directed thrombolysis needs special facilities such as radiological intervention room or C-arm radiological equipment, and it also needs trained personnel to operate the radiological machines. In some cases, the patients cannot undergo radiological intervention due to impaired renal function or allergy to radio-contrast dye. So, catheter-directed thrombolysis is not always available for all patients. Sometimes, surgical thromboembolectomy may be the only option for the patients with acute limb ischemia, when anticoagulation alone is ineffective and catheter-directed thrombolysis is not 
applicable. The portable handheld vascular Doppler device can be used for measuring arterial pulses without any specific restriction or medical hazard. ${ }^{6}$ In this study, we investigated whether limbs could be salvaged if at least one ankle artery was revascularized by surgical thromboembolectomy. During the operation, the handheld vascular Doppler device was used for detecting arterial pulses at ankle, and completion angiography or radiological intervention was not done. We performed surgical thromboembolectomy via the below-knee popliteal artery and observed the clinical result.

\section{Methods}

Between March 1, 2011, and February 28, 2012, we treated 18 acutely ischemic limbs in 14 consecutive patients (10 male and 4 female) who presented at our hospital. All consecutive patients, who were diagnosed to have arterial occlusion by computed tomography (CT) angiography, were included in this study, and there were no inclusion or exclusion criteria. The differentiation between embolic and thrombotic occlusion is based on clinical features and CT angiographic findings. ${ }^{7}$ Embolic occlusion was suggested by the presence of clinical symptoms such as arrhythmia, sudden onset, severe signs and symptoms, no history of claudication or rest pain, no risk factors for peripheral vascular disease, normal contralateral pulse exam, and no physical findings of chronic limb ischemia. Whereas, the thrombotic occlusion was suggested by the clinical findings of no arrhythmia, sudden or slower onset, less severe signs and symptoms, history of claudication or rest pain, risk factors for peripheral vascular disease, abnormal contralateral pulse exam, and physical findings of chronic limb ischemia. The vascular occlusion that has the characteristics of both embolic and thrombotic occlusion is regarded as thromboembolic occlusion. The final differentiation was based on clinical symptoms and CT angiographic findings. This study was approved by the IRB of Haeundae Paik Hospital, Inje University, and written consent was obtained from each patient. The age of the patients ranged from 36 to 83 (mean, 63) years. All 14 patients underwent surgical thromboembolectomy via the popliteal artery below the knee, under general anesthesia.

In this study, even for isolated common femoral arterial lesion, popliteal approach was adopted to avoid the distal embolization of the fragments of thromboemboli into the below-knee arteries during the surgical manipulation of the common femoral artery.

The incision was performed at the medial calf, $1 \mathrm{~cm}$ behind the posterior border of the tibia. The crural fascia was incised $1 \mathrm{~cm}$ posterior to the tibia, and the popliteal artery was exposed below the knee between the space made by the soleus, gastrocnemius, and semitendinosus muscles. We used Fogarty catheters sizes 2 and $3 \mathrm{Fr}$ for distal thromboembolectomy, and sizes 4 and 5 Fr for proximal thromboembolectomy. Distal thromboembolectomy was performed first, and the procedure was stopped once the back flow blood was bright red in color and no more thromboemboli could be removed with the Fogarty catheter. After completion of the distal procedure, the distal arteries were filled with a heparin solution and clamped to prevent embolization from the proximal arteries during proximal thromboembolectomy. The proximal thromboembolectomy was continued until the appearance of strong active bleeding from the proximal artery and until no further thromboemboli could be removed via the Fogarty catheter. After the completion of thromboembolectomy, the popliteal artery was closed either by primary suture or by patch angioplasty using the branch of autogenous saphenous vein. After closing the arteriotomy, arterial pulses at the ankles were measured intraoperatively using a handheld vascular Doppler device (bidirectional Doppler ES-100V3; Hadeco Inc., Kawasaki, Japan). Measurements were made at the dorsalis pedis, posterior tibial, and peroneal arteries of the ankle. The handheld vascular Doppler device detects the frequency shift, amplifies it, and sends it to speakers for audible interpretation. ${ }^{8,9}$ The normal waveform pattern of Doppler signal appearing on the LCD panel of the device typically is triphasic or biphasic, but changes into monophasic when a significant stenosis is present upstream. In this study, the positive pulse was defined when pulse sound was audible and Doppler signal was detected on the LCD panel of the device regardless of its waveform pattern. We stopped surgical thromboembolectomy when a pulse was detected on at least one ankle artery after closing the arteriotomy. If there was no pulse detected, the closed arteriotomy was reopened and the thromboembolectomy was repeated. Then, the progress of arterial revascularization was observed by daily measurements of arterial pulses at the ankle using the handheld vascular Doppler device. In this study, CT angiography was routinely performed for all patients pre- and postoperatively.

Even though limb salvage was successful, CT angiography was routinely performed at 1 week postoperatively to detect any newly developed thromboembolic lesion or vessel change (-Fig. 1).

Anticoagulation therapy with heparin and warfarin was continued during the whole period of treatment. Heparinization was started immediately after the diagnosis of acute limb ischemia. Initially, $80 \mathrm{U} / \mathrm{kg}$ of unfractionated heparin was injected intravenously, and continuous intravenous heparinization was performed keeping the activated partial thromboplastin time (aPTT) between 60 and 100 seconds. Warfarin was administered orally and overlapped the heparin treatment. Heparin was stopped when the international normalized ratio (INR) reached levels between 2.0 and 2.5. Aspirin was also given. For patients with acute limb ischemia and heart problems, warfarin was continuously administered during the whole period of follow-up keeping the INR levels between 2.0 and 2.5. However, for those with no heart disease, warfarin was stopped after 6 months of follow-up and aspirin was given continuously.

\section{Results}

Of the 14 patients, 10 (71\%) had heart disease: 8 of them (57\%) had atrial fibrillation and 2 (14\%) had a history of previous myocardial infarction, 2 and 4 years earlier, respectively. Among the 10 patients with heart disease, 3 patients (21\%) also had hypertension, 2 (14\%) had hypertension and diabetes 

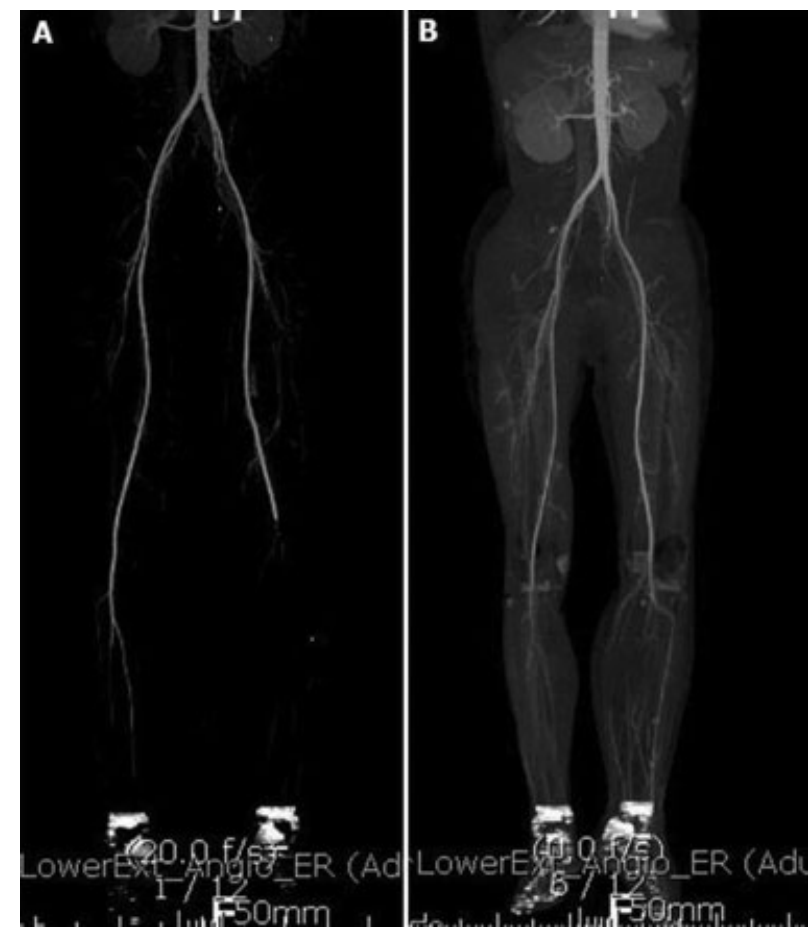

Fig. 1 Preoperative and postoperative CT angiography of a representative patient with embolic occlusion. (a) Preoperative CT angiography shows complete occlusion of left popliteal artery with poor collaterals. (b) Postoperative CT angiography shows the restoration of flow at left popliteal and anterior tibial arteries.

mellitus (DM) simultaneously, and 1 (7\%) had coexisting DM. Among the remaining 4 patients without heart disease, 1 had history of surgery for stomach cancer 6 years earlier, and 1 had a history of ureteral stone; the remaining 2 patients had no identifiable disease. There were 10 patients (71\%) with embolic, 2 (14\%) with thrombotic, and 2 (14\%) with thromboembolic occlusions. Ten patients had unilateral occlusion, and 4 had bilateral occlusion, presenting a total of 18 occluded limbs. The most common anatomical site of arterial occlusion was the left popliteal artery ( 5 limbs, $28 \%$ ), followed by the right common femoral artery (4 limbs, 22\%), the left common femoral artery (4 limbs, 22\%), the right superficial femoral artery ( 2 limbs, $11 \%$ ), the right popliteal artery ( 2 limbs, $11 \%$ ), and the left superficial femoral artery (1 limb, 6\%). The duration of ischemic symptoms varied between 4 and 120 hours. By the clinical staging of acute limb ischemia, ${ }^{4}$ three patients initially presented with class I symptoms (viable), eight with class IIA (marginally threatened), two with class IIB (immediately threatened), and one with class III (irreversible) symptoms. An 82-year-old man with unilateral occlusion and symptom duration of 120 hours showed class III lesion on his right foot, and eventually underwent below-knee amputation. A 77-year-old woman with bilateral occlusion died 6 days after surgery ( $\mathbf{- T a b l e ~} \mathbf{1}$ ). The decision to perform fasciotomy was based on the clinical symptoms including pain and paresthesia in the distal extremity. ${ }^{10}$ But there was no patient who was indicated for fasciotomy. Initially, we were unable to detect the ankle pulse by handheld vascular Doppler device in any of the patients. Immediately after surgical thromboembolectomy, pulses were detected at the dorsalis pedis in 3 out of 18 limbs, at the peroneal arteries in 10 out of 18 limbs, and at the posterior tibial artery in 7 out of 18 limbs. The total revascularization rate at the 3 arteries below the knee, immediately after surgical thromboembolectomy, was $37 \%$ (20 out of 54 arteries). After 1 week from the surgery, viable pulses were detected by the handheld vascular Doppler device in the remaining 12 patients on two or more

Table 1 Demographics and clinical characteristics of the patients

\begin{tabular}{|c|c|c|c|c|c|c|c|c|c|c|c|c|c|c|}
\hline $\begin{array}{l}\text { Patient } \\
\text { number }\end{array}$ & 1 & 2 & 3 & 4 & 5 & 6 & 7 & 8 & 9 & 10 & 11 & 12 & 13 & 14 \\
\hline Sex & $M$ & $\mathrm{~F}$ & $M$ & $M$ & $M$ & $M$ & $M$ & $\mathrm{~F}$ & $M$ & $\mathrm{~F}$ & $M$ & $M$ & $\mathrm{~F}$ & $M$ \\
\hline Age (y) & 75 & 77 & 49 & 40 & 49 & 36 & 62 & 83 & 72 & 81 & 46 & 69 & 64 & 82 \\
\hline $\begin{array}{l}\text { Causes of } \\
\text { occlusion }\end{array}$ & TE & $\mathrm{T}$ & $\mathrm{E}$ & $E$ & $E$ & $E$ & $\mathrm{E}$ & $E$ & $\mathrm{~T}$ & $E$ & $E$ & $E$ & $E$ & $\mathrm{TE}$ \\
\hline $\begin{array}{l}\text { Site of } \\
\text { obstruction }\end{array}$ & $\begin{array}{l}\text { R.P } \\
\text { L.P } \\
\text { (bilat) } \\
\end{array}$ & $\begin{array}{l}\text { R.P } \\
\text { L.SFA } \\
\text { (bilat) } \\
\end{array}$ & L.P & $\begin{array}{l}\text { R.CFA } \\
\text { L.CFA } \\
\text { (bilat) }\end{array}$ & L.P & L.P & R.CFA & L.P & R.SFA & R.SFA & L.CFA & L.CFA & $\begin{array}{l}\text { R. CFA } \\
\text { L.CFA } \\
\text { (bilat) }\end{array}$ & R.CFA \\
\hline $\begin{array}{l}\text { Symptom } \\
\text { duration (h) }\end{array}$ & 48 & 8 & 4 & 6 & 24 & 72 & 7 & 12 & 18 & 26 & 8 & 6 & 6 & 120 \\
\hline $\begin{array}{l}\text { Clinical } \\
\text { stage }\end{array}$ & IIA & IIA & 1 & IIA & IIA & IIB & $I \mathrm{~A}$ & 1 & IIA & IIB & IIA & IIA & 1 & III \\
\hline $\begin{array}{l}\text { Heart } \\
\text { disease }\end{array}$ & $\mathrm{AF}$ & $\mathrm{Ml}$ & & & $\mathrm{AF}$ & & & $\mathrm{AF}$ & $\mathrm{Ml}$ & $\mathrm{AF}$ & $\mathrm{AF}$ & $\mathrm{AF}$ & $\mathrm{AF}$ & $\mathrm{AF}$ \\
\hline $\begin{array}{l}\text { Other } \\
\text { disease }\end{array}$ & & $\mathrm{HT}, \mathrm{DM}$ & & $\begin{array}{l}\text { History } \\
\text { ureteral } \\
\text { stone }\end{array}$ & $\mathrm{HT}$ & & $\begin{array}{l}\text { History } \\
\text { stomach } \\
\text { cancer }\end{array}$ & & DM & HT & & $\mathrm{HT}$ & & $\begin{array}{l}\text { HT, } \\
\text { DM }\end{array}$ \\
\hline
\end{tabular}

Abbreviations: AF, atrial fibrillation; bilat, bilateral; CFA, common femoral artery; clinical stage: class I (viable), class IIA (marginally threatened), class IIB (immediately threatened), class III (irreversible); DM, diabetes mellitus; E, embolism; F, female; h, hour; HT, hypertension; L, left; M, male; MI, previous myocardial infarction; N, no; P, popliteal artery; R, right; SFA, superficial femoral artery; T, thrombosis; TE, thromboembolic; Y, yes. 
Table 2 Recovery of arterial pulses at the ankles

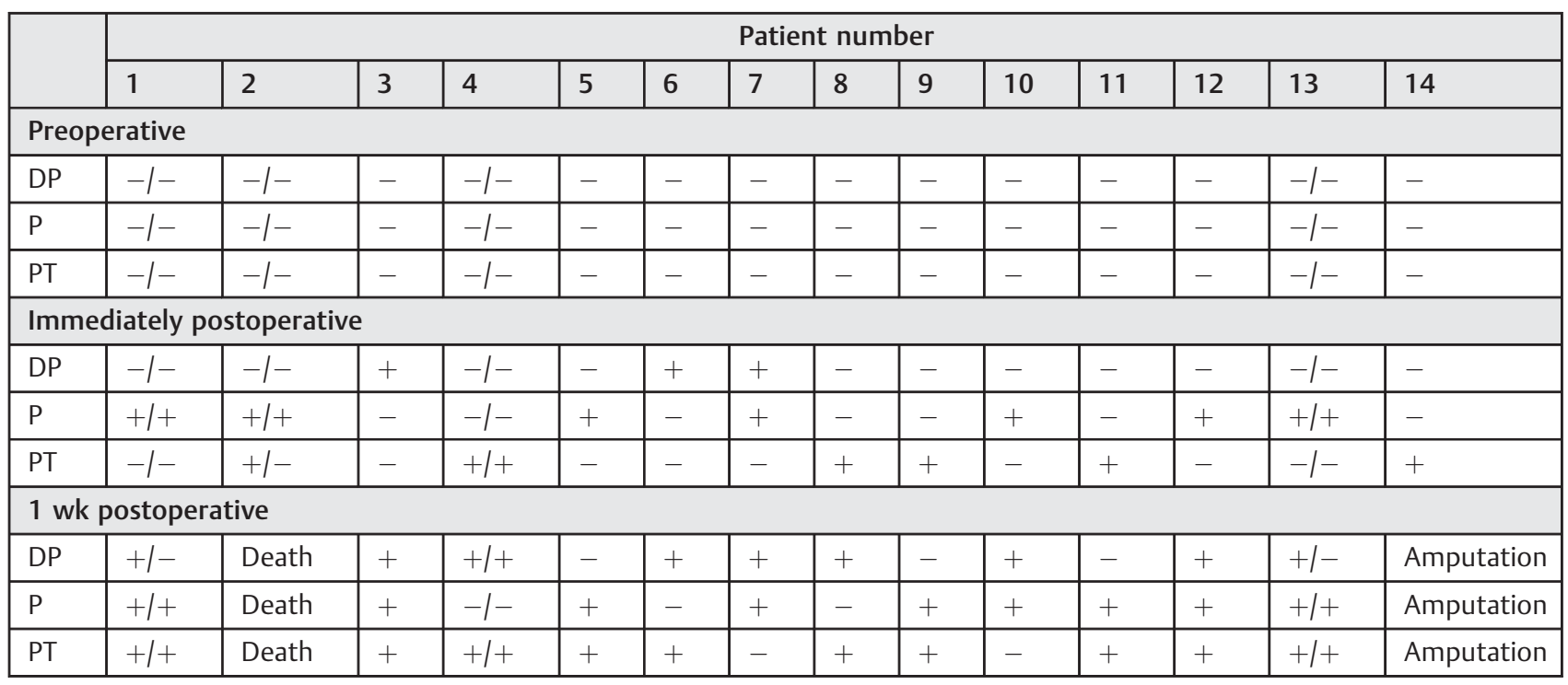

Abbreviations: DP, dorsalis pedis artery (anterior tibial artery); P, peroneal artery; PT, posterior tibial artery; wk, week.

Note: Results show the presence $(+)$ or absence $(-)$ of ankle pulse (right/left) as measured by the handheld vascular Doppler device (bidirectional Doppler ES-100V3; Hadeco Inc., Kawasaki, Japan).

ankle arteries. Leg pain improved markedly in the 12 patients, and there was no tissue loss in their feet. We detected pulses in 10 out of 15 limbs at the dorsalis pedis, 11 out of 15 limbs at the peroneal artery, and 13 out of 15 limbs at the posterior tibial artery. The total revascularization rate 1 week after surgery and anticoagulation therapy was 76\% (34 out of 45 arteries) (-Table 2). All 15 limbs in the 12 patients were salvaged successfully, and during the 6 to 18 months of follow-up, there was no residual complication or tissue loss in the legs.

\section{Discussion}

In this study, pure thrombotic occlusion was found in $14 \%$ of the patients. Whereas, embolic and thromboembolic occlusions were present in $86 \%$ of the patients, and heart disease was present in $71 \%$ of the patients. These findings suggest that acute limb ischemia is closely associated with heart disease, and coincides with the previous reports that heart is the major source of emboli in acute limb ischemia. ${ }^{11,12}$ Acute limb ischemia requires prompt treatment, because one of the negative predictive factors for limb salvage is prolonged duration from symptom onset to surgery. ${ }^{13}$ In this study, an 82-year-old man with a 120-hour of symptom duration finally underwent amputation. In general, patients with class III (irreversible) symptom are not the candidates for revascularization surgery because there are few chances of recovery of viable tissue and an associated high risk of reperfusion injury. ${ }^{4}$ However, the 82 -year-old man had mixed class I and II lesions on his right leg, and the class III lesion was limited to a small portion of his right foot. So, we considered the risk of reperfusion injury to be low, and performed surgical thromboembolectomy. One week after the operation, the patient's right leg above the ankle became warm and pinkish, following which he underwent below-knee amputation without any complication. A 77-year-old woman with bilateral occlusion had suffered from atrial fibrillation, hypertension, and poorly controlled DM. Although revascularization by surgical thromboembolectomy was successful, she died 6 days after surgery due to poor general health and subsequent multiorgan failure. She had also reported transient myoglobinuria and hyperkalemia after surgery, indicating the presence of coexistent reperfusion injury as an additional cause of death.

If ankle artery is adequately thrombectomized but spastic after surgical thromboembolectomy using Fogarty catheter, the Doppler exam may result in negative outcomes.

However, because we tried to investigate the minimally required conditions to finish the operation, we decided to stop the surgical thromboembolectomy when a pulse was detected by a handheld Doppler device on at least one ankle artery after closing the arteriotomy regardless of the real number of thrombectomized ankle arteries.

Immediately after surgical thromboembolectomy, we observed the highest revascularization rate at the peroneal artery (56\%), compared with the posterior tibial (39\%) or dorsalis pedis (17\%) arteries. This may be due to the relatively straight angle of the peroneal artery in relation to the popliteal and tibioperoneal trunk, which might facilitate easier guiding of the Fogarty catheter during thromboembolectomy. Approach through the common femoral artery is a frequently used method for surgical thromboembolectomy. ${ }^{14}$ But in our cases, we attempted the below-knee popliteal approach. We postulated several advantages of the below-knee popliteal approach. First, distal thromboembolectomy is easier due to the shorter length of the distal arteries. Second, proximal thromboembolectomy is more efficient due to the longer length of the proximal occluded artery that is aided by blood pressure in removing clots during surgical thromboembolectomy. Third, 
back flow of blood is therapeutically more significant due to the presence of fewer collateral vessels in the calf compared with the thigh, in which there are many collateral vessels adjacent to the deep femoral artery. In our 14 patients with acute limb ischemia, except for 1 dead and 1 amputated, all the remaining 12 patients showed 100\% limb salvage in their 15 limbs. This result indicates that the below-knee popliteal approach is a successful method in terms of limb salvage. However, there is no comparative data to substantiate this claim. So further study on below-knee popliteal approach comparing with other approaches such as common femoral or above-knee popliteal approaches is needed.

\section{Conclusion}

For patients with acute limb ischemia, successful limb salvage can be achieved by the revascularization of at least one ankle artery by surgical thromboembolectomy with concomitant anticoagulation therapy. Handheld vascular Doppler device is a useful instrument for intraoperative measurement and postoperative monitor of the ankle pulses. Moreover, below-knee popliteal approach is an effective method in terms of limb salvage, and is worth for further study compared with other approaches.

\section{Acknowledgment}

This work was supported by the 2012 Inje University research grant.

\section{References}

1 Results of a prospective randomized trial evaluating surgery versus thrombolysis for ischemia of the lower extremity. The STILE trial. Ann Surg 1994;220(3):251-266, discussion 266-268
2 Ouriel K, Veith FJ, Sasahara AA; TOPAS Investigators. Thrombolysis or peripheral arterial surgery: phase I results. J Vasc Surg 1996;23 (1):64-73, discussion 74-75

3 Comerota AJ, Gravett MH. Do randomized trials of thrombolysis versus open revascularization still apply to current management: what has changed? Semin Vasc Surg 2009;22(1):41-46

4 Rutherford RB. Clinical staging of acute limb ischemia as the basis for choice of revascularization method: when and how to intervene. Semin Vasc Surg 2009;22(1):5-9

5 Carlson GA, Hoballah JJ, Sharp WJ. Surgical thrombectomy: current role in thromboembolic occlusions. Tech Vasc Interv Radiol 2003; $6(1): 14-21$

6 Blankensteijn JD, Gertler JP, Brewster DC, Cambria RP, LaMuraglia GM, Abbott WM. Intraoperative determinants of infrainguinal bypass graft patency: a prospective study. Eur J Vasc Endovasc Surg 1995;9(4):375-382

7 O'Connell JB, Quiñones-Baldrich WJ. Proper evaluation and management of acute embolic versus thrombotic limb ischemia. Semin Vasc Surg 2009;22(1):10-16

8 Bascom PA, Johnston KW, Cobbold RS, Ojha M. Defining the limitations of measurements from Doppler spectral recordings. J Vasc Surg 1996;24(1):34-44, discussion 44-45

9 Stewart AH, Lucas A, Smith FC, Baird RN, Lamont PM. Pre-operative hand-held Doppler run-off score can be used to stratify risk prior to infra-inguinal bypass surgery. Eur J Vasc Endovasc Surg 2002; 23(6):500-504

10 Jensen SL, Sandermann J. Compartment syndrome and fasciotomy in vascular surgery. A review of 57 cases. Eur J Vasc Endovasc Surg 1997;13(1):48-53

11 Abbott WM, Maloney RD, McCabe CC, Lee CE, Wirthlin LS. Arterial embolism: a 44 year perspective. Am J Surg 1982;143(4): 460-464

12 Walker TG. Acute limb ischemia. Tech Vasc Interv Radiol 2009; 12(2):117-129

13 Morris-Stiff G, D’Souza J, Raman S, Paulvannan S, Lewis MH. Update experience of surgery for acute limb ischaemia in a district general hospital-are we getting any better? Ann R Coll Surg Engl 2009;91(8):637-640

14 Henke PK. Contemporary management of acute limb ischemia: factors associated with amputation and in-hospital mortality. Semin Vasc Surg 2009;22(1):34-40 